\title{
Opicapone in Parkinson's Disease: Real-World Data from a Portuguese Center
}

\author{
Filipa Meira-Carvalho ${ }^{a}$ Jorge Diogo Da Silva ${ }^{b, c}$ Margarida Rodrigues ${ }^{a}$ \\ ${ }^{a}$ Department of Neurology, Hospital of Braga, Braga, Portugal; b Life and Health Sciences Research Institute (ICVS), \\ University of Minho, Braga, Portugal; 'ICVS/3B's - PT Government Associate Laboratory, Braga/Guimarães, Portugal
}

Dear Editor,

Opicapone is a novel treatment option for patients with Parkinson's disease (PD) and motor fluctuations, with proven efficacy and safety in clinical trials [1-4]. Nevertheless, it is fundamental to capture the benefit and risk after a product has received regulatory approval and increase its role in health-care decisions based on realworld data.

We carried out a retrospective study to evaluate the therapeutic benefits, safety, and tolerability of opicapone in day-to-day clinical practice. All 2019 clinical registries from movement disorder outpatient visits to the Neurology Department of our center were reviewed. All the patients with a PD diagnosis that initiated opicapone from January to December 2019 were included. All these patients had been managed by one of the three movement disorder specialists of our center.

We documented sociodemographic (age and sex) and historical relevant data (total daily levodopa equivalent dose (LED), time since PD diagnosis, time since motor fluctuations, and time under opicapone therapy). Before and after introducing opicapone, levodopa and other adjunct PD therapies were recorded as the daily LED (Table 1), by using described conversion factors [1]. Addi- tionally, after opicapone initiation, patients' and clinicians' perception of symptom improvement was objectified by the Global Impression of Change scale (PGI-C and CGI-C, respectively), with scores ranging from 1 (very much improved) to 7 (very much worse) [2]. Changes in total daily LED, adverse events (AEs), dropouts, and reasons for discontinuation were also evaluated. Related quantitative data are presented as mean \pm standard deviation.

Opicapone at a dosage of $50 \mathrm{mg}$ per day was initiated in 35 patients: the mean age was $71.2 \pm 8.9$ years, and 22 (63\%) were male (Table 1). On average, time since PD diagnosis was $7.9 \pm 3.6$ years, and time since motor fluctuations was $2.8 \pm 1.9$ years.

Twenty-six of these patients had at least one revaluation appointment. In these, we observed a perception of improvement of PD symptoms soon after initiation (3.5 \pm 1.9 months after the beginning of the treatment, on average), by both clinicians and patients: CGI-C $2.86 \pm 0.56$ points and PGI-C $2.88 \pm 1.07$ points.

The introduction of opicapone led to an overall significant baseline LED reduction, which was observed in 13 of the reevaluated patients $(50 \%)$, with a mean decrease of $226.5 \pm 125.6 \mathrm{mg}$ (1 sample Student's $t$ test with
Correspondence to:

Filipa Meira-Carvalho, ana.filipa.carvalho@ @b.min-saude.pt 
Table 1. Summary of patients' characteristics and study variables

\begin{tabular}{|c|c|c|}
\hline & PD patients & $N$ \\
\hline Men & $22(63)$ & \\
\hline Age, years & $71.2 \pm 8.9$ & \\
\hline Time since PD diagnosis, years & $7.9 \pm 3.6$ & 35 \\
\hline Time since motor fluctuations, years & $2.8 \pm 1.9$ & \\
\hline Total daily LED at baseline, $\mathrm{mg}$ & $1,005.7 \pm 442.7$ & \\
\hline \multicolumn{3}{|l|}{ Levodopa treatment at baseline } \\
\hline Levodopa and benserazide & $15(58)$ & \\
\hline Levodopa and carbidopa & $11(50)$ & \\
\hline Controlled-release levodopa & $15(58)$ & \\
\hline Combination of controlled-release levodopa and immediate-release levodopa & $15(58)$ & \\
\hline \multicolumn{3}{|l|}{ Adjunct medications at baseline $\mathrm{a}^{\mathrm{a}}$} \\
\hline Amantadine & $7(27)$ & \\
\hline Catechol-o-methyl transferase inhibitor ${ }^{\mathrm{b}, \mathrm{c}}$ & $3(12)$ & \\
\hline Dopamine agonists ${ }^{\mathrm{b}}$ & $20(77)$ & \\
\hline Monoamine oxidase B inhibitors ${ }^{\mathrm{b}}$ & $7(27)$ & \\
\hline Time under opicapone, months & $9.04 \pm 3.96$ & 26 \\
\hline PGI-C score, points ${ }^{\mathrm{d}}$ & $2.88 \pm 1.07$ & \\
\hline CGI-C score, points ${ }^{\mathrm{d}}$ & $2.86 \pm 0.56$ & \\
\hline Total daily LED reduction & $13(50)$ & \\
\hline Mean decrease, mg & $226.5 \pm 125.6$ & \\
\hline Adverse events & $19(73)$ & \\
\hline Dyskinesia & $12(46)$ & \\
\hline Leading to discontinuation & $6(23)$ & \\
\hline Dyskinesia & $3(12)$ & \\
\hline Hallucinations & $1(4)$ & \\
\hline Orthostatic hypotension & $1(4)$ & \\
\hline Confusional state & $1(4)$ & \\
\hline
\end{tabular}

$N(\%)$ or mean \pm standard deviation. PD, Parkinson's disease; LED, levodopa equivalent dose; PGI-C, Patient Global Impression of Change; CGI-C, Clinical Global Impression of Change. ${ }^{\text {a }}$ Patients could receive more than one adjunct treatment. ${ }^{\mathrm{b}}$ Catechol-o-methyl transferase inhibitor was entacapone $(n=3)$; dopamine agonists included pramipexole $(n=2)$, ropinirole $(n=14)$, and rotigotine $(n=4)$; monoamine oxidase B inhibitors included oral selegiline $(n=3)$ and rasagiline $(n=4) .{ }^{\mathrm{c}}$ While these 3 patients had entacapone at baseline, their baseline LED reduction after opicapone initiation was also accounted by levodopa and dopamine agonist adjustments. ${ }^{\mathrm{d}}$ Scores range from 1 to 7 , with 1 indicating very much improved; 2 , much improved, 3 , minimally improved; 4 , no change; 5 , minimally worse; 6 , much worse; and 7 , very much worse.

comparison to a value of $0: t(25)=3.994, p=0.001, d=$ $0.783)$. In the majority of these $(n=8 ; 62 \%)$, baseline LED reduction was initiated as soon as opicapone was introduced. In the remaining 5 patients, baseline LED reduction was performed, on average, $5.2 \pm 3.2$ months later, and aimed to overcome the AEs related to opicapone initiation (mainly dyskinesia - see below). None of these 13 patients had to discontinue opicapone due to AEs.

Regarding adverse effects, 19 of the 26 patients who started opicapone experienced at least one $\mathrm{AE}(73 \%)$, with dyskinesia being the most common $(n=12 ; 46 \%)$. Orthostatic hypotension $(n=4 ; 15 \%)$, constipation $(n=$ $3 ; 12 \%)$, dizziness $(n=3 ; 12 \%)$, hallucinations $(n=2 ; 8 \%)$, dry mouth $(n=1 ; 4 \%)$, and confusional state $(n=1 ; 4 \%)$ were also reported. Most dyskinetic events occurred in patients already experiencing dyskinesia at baseline $(n=$ $10 ; 38 \%)$. Five (19\%) patients reported more than one AE.

Opicapone was discontinued in 6 patients due to AEs (23\%), due to their severity and patient preference. The most common event leading to discontinuation was dyskinesia $(n=3 ; 12 \%)$; other dopaminergic events, namely, hallucinations $(n=1 ; 4 \%)$ and orthostatic hypotension $(n=1 ; 4 \%)$, as well as confusional state $(n=1 ; 4 \%)$ were the other reasons reported. All these 6 patients belonged to a group of 13 patients who had no baseline LED reduction, whether regarding levodopa or other PD drugs. In 
contrast, as described above, none of the 13 patients who had baseline LED reduction had to discontinue opicapone.

In line with data from clinical trials [3-7], opicapone was globally beneficial in patients with advanced PD and motor fluctuations under levodopa and other adjunct PD therapies. The therapeutic impact of this drug was soon perceived by both clinicians and patients. Additionally, total daily LED reduction was largely significant and possible in half of the reevaluated patients after opicapone initiation, positioning this compound as a strong contender for adjunctive therapy of motor fluctuations in PD.

Nevertheless, $23 \%$ of patients had to discontinue opicapone due to AEs. Dyskinesia, reflecting greater dopaminergic availability, was the most common $\mathrm{AE}$ and the leading cause of discontinuation - in fact, $25 \%$ of patients with dyskinesia suspended opicapone. However, these dyskinetic events were more frequent in patients with previously known dyskinesia, and several were successfully managed by further adjustments of dopaminergic therapy. Other AEs were uncommon, further reinforcing the benefit and safety of opicapone in advanced PD.

This study has some limitations such as the modest sample size, which is in part due to the currently short period of commercialization of opicapone after its approval. Additionally, as a retrospective study, data regarding other clinical variables such as differences in motor disability (specifically assessed by MDS-UPDRS-III) and motor complications (by MDS-UPDRS-IV) were not available and should be assessed in future studies. Nevertheless, we consider that impressions of improvement, both by patients and movement disorder specialists, are a strong and relevant clinical indicator. In summary, we provided clinical practice data regarding the use of a recently approved drug for advanced PD, in the hope of optimizing the management of these patients.

\section{Statement of Ethics}

This retrospective review of patient data did not require ethical approval in accordance with local/national guidelines. Written informed consent from participants was not required in accordance with local/national guidelines.

\section{Conflict of Interest Statement}

Filipa Meira Carvalho received financial support to attend meetings from Zambon. Margarida Rodrigues received financial support to speak or attend meetings from Bial and Zambon.

\section{Funding Sources}

No funding was provided for this work.

\section{Author Contributions}

Research project: conception, organization, and execution Filipa Meira Carvalho, Jorge Diogo Da Silva, and Margarida Rodrigues. Statistical analysis: design, review, and critique - Filipa Meira Carvalho, Jorge Diogo Da Silva, and Margarida Rodrigues. Statistical analysis: execution - Filipa Meira Carvalho and Jorge Diogo Da Silva. Manuscript: writing of the first draft - Filipa Meira Carvalho. Manuscript: review and critique - Filipa Meira Carvalho, Jorge Diogo Da Silva, and Margarida Rodrigues.

\section{References}

1 Tomlinson CL, Stowe R, Patel S, Rick C, Gray $\mathrm{R}$, Clarke CE. Systematic review of levodopa dose equivalency reporting in Parkinson's disease. Mov Disord. 2010;25(15):2649-53.

2 Guy W. Clinical global impressions. In. National Institute of Mental Health, editor. ECDEU assessment manual for psychopharmacology. Rockville, MD: Department of Health, Education, and Welfare; 1976. p. 218-222.

3 Ferreira JJ, Lees A, Rocha J-F, Poewe W, Rascol O, Soares-da-Silva P. Long-term efficacy of opicapone in fluctuating Parkinson's dis- ease patients: a pooled analysis of data from two phase 3 clinical trials and their open-label extensions. Eur J Neurol. 2019;26:953-60.

4 Ferreira JJ, Lees A, Rocha J-F, Poewe W, Rascol O, Soares-da-Silva P. Opicapone as an adjunct to levodopa in patients with Parkinson's disease and end-of-dose motor fluctuations: a randomised, double-blind, controlled trial. Lancet Neurol. 2016;15:154-65.

5 Lees AJ, Ferreira J, Rascol O, Poewe W, Rocha JF, McCrory M, et al. Opicapone as adjunct to levodopa therapy in patients with Parkinson disease and motor fluctuations: a randomized clinical trial. JAMA Neurol. 2017;74(2):197206.

6 Ferreira JJ, Lees AJ, Poewe W, Rascol O, Rocha JF, Keller B, et al. Effectiveness of opicapone and switching from entacapone in fluctuating Parkinson disease. Neurology. 2018; 90(21):e1849-57.

7 Castro Caldas A, Teodoro T, Ferreira JJ. The launch of opicapone for Parkinson's disease: negatives versus positives. Expert Opin Drug Saf. 2018;17:331-7. 